\title{
LDPC Code Design for the Two-User Gaussian Multiple Access Channel
}

\author{
Shahrouz Sharifi, Student Member, IEEE, A. Korhan Tanc, Member, IEEE, and Tolga M. Duman, Fellow, IEEE
}

\begin{abstract}
We study code design for two-user Gaussian multiple access channels (GMACs) under fixed channel gains and under quasi-static fading. We employ low-density parity-check (LDPC) codes with BPSK modulation and utilize an iterative joint decoder. Adopting a belief propagation (BP) algorithm, we derive the PDF of the log-likelihood-ratios (LLRs) fed to the component LDPC decoders. Via examples, it is illustrated that the characterized PDF resembles a Gaussian mixture (GM) distribution, which is exploited in predicting the decoding performance of LDPC codes over GMACs. Based on the GM assumption, we propose variants of existing analysis methods, named modified density evolution (DE) and modified extrinsic information transfer (EXIT). We derive a stability condition on the degree distributions of the LDPC code ensembles and utilize it in the code optimization. Under fixed channel gains, the newly optimized codes are shown to perform close to the capacity region boundary outperforming the existing designs and the off-the-shelf point-to-point (P2P) codes. Under quasi-static fading, optimized codes exhibit consistent improvements upon the P2P codes as well. Finite block length simulations of specific codes picked from the designed ensembles are also carried out and it is shown that optimized codes perform close to the outage limits.
\end{abstract}

Index Terms-Low-density parity-check codes, code design, multiple access channels, iterative decoding, Gaussian mixture, common outage capacity.

\section{INTRODUCTION}

A GAUSSIAN multiple access channel (GMAC) in its simplest form consists of two users communicating with one receiver in the presence of additive white Gaussian noise (AWGN). The capacity region of the two-user GMAC has been completely characterized. The corner points of the capacity region can be achieved via single user decoding, hence via time sharing one can achieve the points in between. It is also shown that any rate pair in the capacity region can be attained utilizing rate splitting or joint decoding without the need for time sharing

\footnotetext{
Manuscript received June 3, 2015; revised October 3, 2015; accepted December 10, 2015. Date of publication December 23, 2015; date of current version April 7, 2016. This work was supported in part by the National Science Foundation under Grant NSF-CCF 1117174, in part by the European Commission under Grant MC-CIG PCIG12-GA-2012-334213, and in part by the Turkish Scientific and Technological Research Council of Turkey (TUBITAK) under Grant 114E601. The associate editor coordinating the review of this paper and approving it for publication was Z. Wang.

S. Sharifi was with the School of Electrical, Computer, and Energy Engineering (ECEE), Arizona State University, Tempe, AZ 85287 USA. He is now with Maxlinear, Carlsbad, CA, USA (e-mail: ssharifi@maxlinear.com).

A. K. Tanc is with the Department of Electrical and Electronics Engineering, Kirklareli University, Kayali 39100, Kirklareli, Turkey (e-mail: korhan.tanc@ kirklareli.edu.tr)

T. M. Duman is with the Department of Electrical and Electronics Engineering, Bilkent University, Bilkent 06800, Ankara, Turkey (e-mail: duman@ee.bilkent.edu.tr).

Digital Object Identifier 10.1109/TWC.2015.2511749
}

[1]. From a practical channel coding perspective, authors in [1], [2] utilize low-density-parity-check (LDPC) codes and implement an iterative joint decoding algorithm achieving rate pairs close to the boundary for a two-user GMAC with equal channel gains.

LDPC codes are powerful linear block codes introduced by Gallager in [3]. While they were forgotten for a long time (except for some sporadic works) presumably due to the complexity of the encoding and decoding schemes, they were reintroduced in the work of MacKay [4] who rediscovered the potential of the linear block codes with sparse parity-check matrices. These codes have been successfully employed for various channels, and promising rates close to the information theoretic limits have been attained. Motivated by their superior performance for different channels and their premise in [1] and [2], in this paper, we explore the problem of LDPC code design for the more general two-user GMAC with BPSK modulation. We consider two scenarios for channel gains: fixed and quasistatic fading. The former scenario suits time-invariant models, while the latter models the scenarios for which the fading is so slow that no matter how long the codeword is, the (random) channel gain remains constant. We do not consider the fast fading scenario, since a similar model is already investigated in [5] for the two-user degraded broadcast channel whose results can be readily applied to the MAC scenario.

LDPC codes exhibit a threshold effect which determines, in terms of the channel parameters, when the decoding error probability can be made arbitrary small. Density evolution (DE) [6] is the primary technique in computing the decoding thresholds. Full implementation of the DE requires extensive calculations, therefore quantized DE [6] is commonly employed in the literature. A similar approach is followed in [7] by employing DE for the two-user GMAC for a joint decoder wherein look-up tables are exploited to update the PDFs of the log-likelihoodratios (LLRs) fed to the component LDPC decoders through the so-called state nodes [2].

Extrinsic information transfer (EXIT) analysis [8] is an alternative to the DE method tracking the evolution of the mutual information between the transmitted bits and the corresponding LLRs exchanged within the decoder. The common assumption in the EXIT analysis, which greatly simplifies the computations, is to consider Gaussian densities for the LLRs. Authors in [5] employ an EXIT analysis to optimize LDPC codes for the two-user degraded broadcast channel utilizing a joint decoder at the better receiver where they adopt a simple linear approximation to update the evolution of the mutual information at the state nodes. The authors in [2] also utilize an EXIT analysis to optimize irregular LDPC codes for the two-user GMAC with 
equal channel gains. Unlike [5], they compute the evolution of the mutual information as the average of the values obtained for two types of state nodes based on the transmitted (coded) bits. Authors in [9] study a similar channel model and adopt an EXIT analysis to design distributed joint source-channel codes. They show for some (simulation) examples that the PDFs of the outgoing LLRs from the state nodes resemble a Gaussian mixture (GM) distribution, however, for simplification, they opt for using the Gaussian assumption.

Motivated by the results of [9], we analytically characterize the PDF of the outgoing LLRs from the state nodes for fixed channel gains and illustrate via examples that the PDF of the outgoing LLRs from the state nodes resembles a GM distribution. Based on this observation, we utilize the GM assumption and modify both the existing DE [7] and EXIT analysis [5] methods. We refer to the new algorithms as the modified DE and the modified EXIT analysis throughout the paper. For the modified DE, the PDFs of the outgoing LLRs from the state nodes are fitted with GM distributions. The parameters of the GM distributions are estimated by employing the expectation maximization (EM) method run over the samples generated via Monte Carlo simulations [10]. The obtained PDFs are then fed to the component LDPC decoders where common method of [11] is adopted to track the evolution of the PDFs exchanged between the check nodes and the variable nodes. For the modified EXIT analysis, the evolution of the mutual information associated with the exchanged LLRs are computed analytically exploiting the GM assumption. Unlike [12], the computations are performed with no limitation on the ratio of the variance to the mean of the PDFs. Considering the fixed channel gains scenario, we incorporate the proposed methods of modified DE and modified EXIT analysis into the LDPC code optimization, and we utilize a random perturbation technique also exploited in [13] for a different problem.

We provide many code design examples in the paper. We demonstrate that our optimized LDPC codes for the case of equal channel gains are shown to improve upon the ones designed in [2]. For the case of unequal channel gains, we show that our optimized codes offer better performance compared to the ones attained via the method of [5]. In addition, we highlight that the optimized codes outperform the point-to-point (P2P) codes designed for binary-input AWGN (BI-AWGN) channels. For the quasi-static fading scenario, we consider the common outage probability [14] as the performance measure. Despite the superior error-rate performance of our methods, the amount of computations prohibits their use in quasi-static fading, hence to simplify the analysis and reduce the amount of computations, we incorporate the simple method of [5] in the code optimization process. We carry out the code design for examples of real and complex channel gains and demonstrate that the newly designed codes consistently improve upon the existing P2P designs. We also perform finite code block length simulations confirming the superiority of the new designs.

The rest of the paper is organized as follows. In Section II, the system model is described and coding/decoding schemes are elaborated. In Section III, the PDF of the outgoing LLRs from the state nodes is computed and a stability condition is derived for the LDPC codes employed for two-user GMACs. In
Section IV, we explain the proposed variants of the DE and the EXIT analysis based on GM assumption. In Section V, we elaborate on the code optimization procedure. In Section VI, numerical examples and simulation results are provided. Finally, Section VII concludes the paper.

\section{System Model AND PReliminaries}

Consider a two-user GMAC where the received signal $Y$ is expressed as

$$
Y=H_{1} X_{1}+H_{2} X_{2}+Z,
$$

where $X_{i}$ represents the signal of the user $i$ with the average power $E\left\{\left|X_{i}\right|^{2}\right\}=1(i=1,2)$, and $Z$ denotes the circularly symmetric complex AWGN with variance $\frac{1}{2}$ per dimension. The average received power of the user $i$ is defined as $P_{i}=$ $\left|H_{i}\right|^{2}$ with $H_{i}$ denoting the channel gain between the user $i$ and the receiver. We consider two scenarios: fixed channel gains and quasi-static fading. For the former case, the channel gains are unchanged throughout the entire transmissions, while in the latter, they are drawn randomly but kept fixed during the transmission of each codeword.

\section{A. Shannon Capacity vs Outage Capacity}

For the case with fixed channel gains, the Shannon capacity of the two-user GMAC is the convex hull of the rate pairs $\left(R_{1}, R_{2}\right)$ characterized as [15]

$$
\begin{aligned}
R_{1} & <I\left(X_{1} ; Y \mid X_{2}\right), \\
R_{2} & <I\left(X_{2} ; Y \mid X_{1}\right), \\
R_{1}+R_{2} & <I\left(X_{1}, X_{2} ; Y\right),
\end{aligned}
$$

over all product distributions $p_{X_{1}}\left(x_{1}\right) \cdot p_{X_{2}}\left(x_{2}\right)$. Under quasistatic fading, reliable transmission is not guaranteed for all the channel realizations, therefore the Shannon capacity is zero. Authors in [14] introduced the common outage capacity region (COCR) computed as

$$
\begin{array}{r}
\operatorname{Pr}\left\{R_{1}<I\left(Y ; X_{1} \mid X_{2}\right)\right\} \geq 1-P_{o}, \\
\operatorname{Pr}\left\{R_{2}<I\left(Y ; X_{2} \mid X_{1}\right)\right\} \geq 1-P_{o}, \\
\operatorname{Pr}\left\{R_{1}+R_{2}<I\left(Y ; X_{1}, X_{2}\right)\right\} \geq 1-P_{o},
\end{array}
$$

over all PDFs $p_{X_{1}}\left(x_{1}\right) \cdot p_{X_{2}}\left(x_{2}\right)$ where $P_{o}$ is called common outage probability.

The rate region in (1) can be calculated analytically for Gaussian signaling [16]. For BPSK signaling, however, numerical calculations are needed. Here, we adopt a grid search method to characterize COCR for BPSK signaling considering real channel gains. The boundary of the COCR can be characterized by solving

$$
\begin{aligned}
\min _{R_{1}, R_{2}} & \left|\tilde{P}_{o}\left(R_{1}, R_{2}\right)-P_{o}\right| \\
\text { s.t. } & 0 \leq R_{1} \leq R_{1_{\max }}, \\
& 0 \leq R_{2} \leq R_{2_{\max }},
\end{aligned}
$$




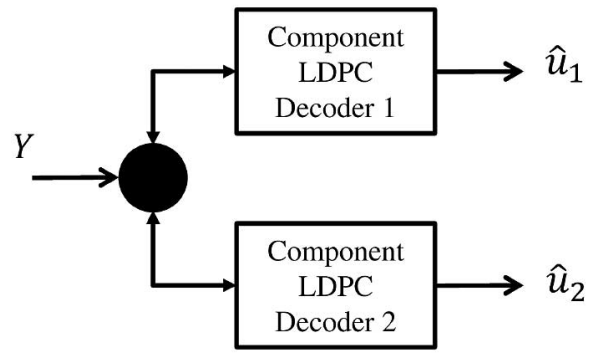

Fig. 1. Block diagram of the joint decoder adopted at the receiver side.

where $\tilde{P}_{o}\left(R_{1}, R_{2}\right)$ is computed as

$$
\begin{aligned}
\tilde{P}_{o}\left(R_{1}, R_{2}\right)=\int_{0}^{\infty} \int_{0}^{\infty} & \mathbb{1}_{\operatorname{COCR}\left(R_{1}, R_{2}, h_{1}, h_{2}\right)} \\
& \times f_{H_{1}}\left(h_{1}\right) f_{H_{2}}\left(h_{2}\right) d h_{1} d h_{2} .
\end{aligned}
$$

The function $\mathbb{1}_{\text {COCR }}$ equals 1 if, for a given $\left(R_{1}, R_{2}, h_{1}, h_{2}\right)$, the rate pairs are inside the conditional rate region (1), otherwise is set to $0 . R_{i_{\max }}$ denotes the capacity of the P2P channel between the user $i$ and the receiver.

\section{B. I.I.D. Channel Adapters}

The decoding analysis of the LDPC codes can be greatly simplified for a symmetric channel by analyzing the behavior of the decoder for the all-zero codeword [6]. A channel is called symmetric if $f_{Y_{i}}\left(y \mid C_{i}=0\right)=f_{Y_{i}}\left(-y \mid C_{i}=1\right)$, where $C_{i}$ and $Y_{i}$ refer to the $i$ th coded bit and the $i$ th channel output, respectively, and $f_{Y_{i}}$ denotes the PDF of $Y_{i}$ conditioned on $C_{i}$. Unlike the case of BI-AWGN channels, the channel symmetry does not hold for multi-user channels in general. To address this issue in our setting, we employ the independent and identically distributed (i.i.d.) channel adapters [17] applied at the transmitter and receiver sides. The idea is to combine each codeword with a random sequence via module- 2 addition prior to transmission and utilize the same set of sequences at the receiver for decoding of each codeword. It should be noted that the i.i.d. channel adapters are employed to simplify the analysis and are not implemented during the actual encoding and decoding processes.

\section{Coding and Decoding Schemes}

At the transmitter sides, the information bits of each user, denoted as $u_{j}(j=1,2)$, are encoded with an LDPC code. The $i$ th encoded bit of $u_{j}$, denoted by $c_{j}(i)$, is modulated using BPSK and sent over the channel as $X_{j}(i)=\left(1-2 c_{j}(i)\right)$. At the receiver side, a joint decoder is utilized wherein a BP based algorithm is adopted to decode the messages in an iterative fashion. As shown in Fig. 1, a joint decoder can be formed by combining two component LDPC decoders which exchange the updated LLRs through the state nodes. Exchange of soft information between the component LDPC decoders can be scheduled serially or in parallel [2]. In serial scheduling only one component decoder is active at a time while under parallel scheduling both run simultaneously. As in [2], we adopt parallel scheduling in the rest of the paper.

\section{Analysis of the JoInt Decoding Algorithm}

We now briefly review the irregular LDPC codes and compute a stability condition on the corresponding code degree distributions used over two-user GMACs. In addition, we elaborate on the computation of the outgoing LLRs from the state nodes and derive the associated PDFs.

Irregular LDPC codes have successfully been employed over various channels [2], [5], [18]. Motivated by their excellent performance, we consider their use over two-user GMACs throughout the paper. Following the notation in [6], an ensemble of irregular LDPC codes $(\lambda, \rho)$ is described by $\lambda(x)=$ $\sum_{i=2}^{d_{v}} \lambda_{i} x^{i-1}$ and $\rho(x)=\sum_{i=2}^{d_{c}} \rho_{i} x^{i-1}$, where $d_{v}$ and $d_{c}$ are the maximum degrees of variable nodes and check nodes, respectively, and the design rate of the code is computed as

$$
r=1-\frac{\sum_{i=2}^{d_{c}} \rho_{i} / i}{\sum_{i=2}^{d_{v}} \lambda_{i} / i}
$$

\section{A. Stability Condition}

Authors in [6] introduced a stability condition analyzing the asymptotic decoding behavior of an LDPC code ensemble used over a BI-AWGN channel. The stability condition is further studied for multi-user scenarios [2], [5], [13], for instance, authors in [2] compute the stability condition for twouser GMACs when channel gains are identical and real. Here, we derive the stability condition conditioned on the channel gains for the general scenario of complex channel gains ${ }^{1}$. For simplicity of the analysis, we follow the approach taken in [13] and assume that the joint decoder is operating at steady state and close to successful decoding. We derive the stability condition for the component LDPC decoder $j$ assuming the other component LDPC decoder has almost decoded its own message, therefore the modified channel output $Y^{\prime}$ [13] is obtained as $Y^{\prime}=H_{j} X_{j}+Z$, which resembles a P2P channel. As a consequence, the LLR received at the $i$ th variable node of component LDPC decoder $j$ is simplified to $L\left(c_{j}(i)\right)=$ $4 \operatorname{Re}\left\{H_{j} Y^{\prime *}\right\}$. Considering the symmetry condition [13], it is easy to show that after applying the channel adapters, $L\left(c_{j}(i)\right)$ is distributed as $\mathcal{N}\left(4\left|H_{j}\right|^{2}, 8\left|H_{j}\right|^{2}\right)$. Hence, results of [6] can be utilized to derive the stability condition, given by

$$
\lambda_{j}^{\prime}(0) \rho_{j}^{\prime}(1)<\exp \left(\left|H_{j}\right|^{2}\right),
$$

where $\lambda_{j}^{\prime}(0)$ denotes the derivative of polynomial $\lambda$ of user $j$ computed at zero. For the quasi-static scenario, $H_{j}$ changes from one codeword to another.

\section{B. Characterization of Outgoing LLRs from State Nodes}

Considering the BP rule at the state nodes, the LLR corresponding to the $i$ th coded bit of the message of user $j$ is computed as

$$
L\left(c_{j}(i)\right)=\log \left(\frac{f_{Y_{i}}\left(y \mid c_{j}(i)=0\right)}{f_{Y_{i}}\left(y \mid c_{j}(i)=1\right)}\right) .
$$

\footnotetext{
${ }^{1}$ The case of real channel gains can be handled similarly.
} 
The update rule (3) is a non-linear operation, therefore existing performance analysis techniques employ look-up tables or numerical methods to evaluate the PDF of the outgoing LLRs from the state nodes. In the following, we derive the PDF of the LLRs analytically. To simplify the analysis, we consider the case of real channel gains. Also, we discard the bit index in the expressions for the ease of the exposition. Without loss of generality, we compute (3) for the LLR sent from a state node to a variable node of component LDPC decoder 1 given by

$$
\begin{aligned}
L & =4 Y\left(H_{1}-H_{2}\right)+\log \left(\frac{\exp \left(4 H_{2}\left(Y-H_{1}\right)\right) \exp (X)+1}{\exp (X)+\exp \left(-4 H_{2}\left(Y+H_{1}\right)\right)}\right) \\
& =B Y^{\prime}+\log \left(\frac{1+A \exp \left(Y^{\prime}+X\right)}{\exp (X)\left(1+A \exp \left(-Y^{\prime}-X\right)\right)}\right)
\end{aligned}
$$

where $A=\exp \left(-4 H_{2} H_{1}\right), Y^{\prime}=4 H_{2} Y, B=\frac{\left(H_{1}-H_{2}\right)}{H_{2}}$, and $X$ denotes the LLR received at the state node from the other component LDPC decoder. Considering i.i.d. channel adapters, $X$ can be written as $t \cdot X^{\prime}$ where $t$ is a random sequence consisting of 1 and -1 with equal probability [17] for user 2 and $X^{\prime}$ denotes the LLR prior to applying the channel adapters. Therefore, the PDF of the random variable $X$ can be obtained as

$$
f_{X}(x)=\frac{1}{2}\left(f_{X^{\prime}}(x)+f_{X^{\prime}}(-x)\right) .
$$

Similarly, $Y$ can be considered as the channel output corresponding to the transmission of all-ones sequence for the user 1 and a sequence with symbols 1 and -1 drawn with equal probability [2] for user 2. Hence, the PDF of $Y^{\prime}$ is computed as

$$
f_{Y^{\prime}}\left(y^{\prime}\right)=\frac{1}{8 H_{2} \sqrt{\pi}}\left(e^{-\left(\frac{y^{\prime}}{4 H_{2}}-H_{1}-H_{2}\right)^{2}}+e^{-\left(\frac{y^{\prime}}{4 H_{2}}-H_{1}+H_{2}\right)^{2}}\right) .
$$

We derive the PDF of $L$ for the equal channel gains and the unequal channel gains, separately.

1) Equal Channel Gains: Consider the transformation

$$
\begin{aligned}
& Z_{1}=\log \left(\frac{1+A \exp \left(Y^{\prime}+X\right)}{\exp (X)\left(1+A \exp \left(-Y^{\prime}-X\right)\right)}\right), \\
& Z_{2}=Y^{\prime},
\end{aligned}
$$

implying

$$
\begin{aligned}
X & =-Z_{1}+\log \left(\frac{1-A \exp \left(Z_{1}-Z_{2}\right)}{1-A \exp \left(Z_{2}-Z_{1}\right)}\right), \\
Y^{\prime} & =Z_{2},
\end{aligned}
$$

where $-|\log (A)|+Z_{2} \leq Z_{1} \leq|\log (A)|+Z_{2}$. Since $L=Z_{1}$, $f_{L}(l)$ is obtained by marginalizing $f_{Z_{1}, Z_{2}}$ over $Z_{2}$, which is given by

$$
\begin{aligned}
f_{L}(l)= & \int_{F}\left|J\left(l, z_{2}\right)\right| f_{Z_{1}, Z_{2}}\left(l, z_{2}\right) d z_{2}, \\
\stackrel{(a)}{=} & \int_{-|\log (A)|}^{|\log (A)|}\left|\frac{A^{2}-1}{\left(A^{2}-2 A \cosh \left(z_{2}^{\prime}\right)+1\right)}\right| \\
& \times f_{X}\left(-l+\log \left(\frac{1-A \exp \left(-z_{2}^{\prime}\right)}{1-A \exp \left(z_{2}^{\prime}\right)}\right)\right) \\
& \times f_{Y^{\prime}}\left(z_{2}^{\prime}+l\right) d z_{2}^{\prime},
\end{aligned}
$$

where (a) follows from the transformation $z_{2}^{\prime}=z_{2}-l$ and $J(.,$.$) is the Jacobian function defined as$

$$
\left|J\left(z_{1}, z_{2}\right)\right|=\left|\begin{array}{ll}
\frac{\partial x}{\partial z_{1}} & \frac{\partial y^{\prime}}{\partial z_{1}} \\
\frac{\partial x}{\partial z_{2}} & \frac{\partial y^{\prime}}{\partial z_{2}}
\end{array}\right| .
$$

2) Unequal Channel Gains: Similar to the previous case, we adopt the random variable transformation

$$
\begin{aligned}
& Z_{1}=\log \left(\frac{1+A \exp \left(X+Y^{\prime}\right)}{\exp (X)\left(1+A \exp \left(-X-Y^{\prime}\right)\right)}\right), \\
& Z_{2}=B Y^{\prime},
\end{aligned}
$$

where

$$
\begin{aligned}
X & =-Z_{1}+\log \left(\frac{1-A \exp \left(Z_{1}-\frac{Z_{2}}{B}\right)}{1-A \exp \left(-Z_{1}+\frac{Z_{2}}{B}\right)}\right), \\
Y^{\prime} & =\frac{Z_{2}}{B},
\end{aligned}
$$

where $-|\log (A)|+\frac{Z_{2}}{B}<Z_{1}<|\log (A)|+\frac{Z_{2}}{B}$. Since $L=$ $Z_{2}+Z_{1}$, it follows that

$$
\begin{aligned}
& f_{L}(l)=\int f_{Z_{1} Z_{2}}\left(l-z_{2}, z_{2}\right) d z_{2} \\
& =\int_{-\left|\frac{B \log (A)}{1+B}\right|}^{\left|\frac{B \log (A)}{1+B}\right|} f_{X}\left(z_{2}-\frac{l}{B+1}\right. \\
& \left.+\log \left(\frac{1-A \exp \left(-z_{2}\left(\frac{B+1}{B}\right)\right)}{1-A \exp \left(z_{2}\left(\frac{B+1}{B}\right)\right)}\right)\right) \\
& \times f_{Y^{\prime}}\left(\frac{z_{2}+\frac{B l}{B+1}}{B}\right)\left|J\left(\frac{l}{B+1}-z_{2}, z_{2}\right)\right| d z_{2},
\end{aligned}
$$

for $B \neq-1$ and

$$
\begin{aligned}
f_{L}(l)= & \int_{-\infty}^{\infty}\left|J\left(l-z_{2}, z_{2}\right)\right| \\
& \times f_{X}\left(z_{2}-l+\log \left(\frac{1-A \exp (l)}{1-A \exp (-l)}\right)\right) \\
& \times f_{Y^{\prime}}\left(\frac{z_{2}}{B}\right) d z_{2},
\end{aligned}
$$

for $B=-1$. Note that at the zeroth iteration, $X=0$, therefore $f_{L}(l)$ for both cases of equal channel gains and unequal channel gains can be computed via the one-to-one transformation from $Y^{\prime}$ to $L$. The computations of (5), (6), and (7), are costly for practical implementations. Therefore, we propose approximating the PDF by a simpler form. In the following, we show that the GM distributions are good candidates for approximating the PDFs. 


\section{GM Approximation}

GM distributions are parametric PDFs represented as a weighted sum of Gaussian component densities given by

$$
f_{L}(l)=\sum_{i=1}^{N} w_{i} \exp \left(-\frac{\left(l-\mu_{i}\right)^{2}}{2 \sigma_{i}^{2}}\right)
$$

where $\mu_{i}, \sigma_{i}^{2}$, and $w_{i}$ denote the mean, the variance, and the mixing proportion of the Gaussian component $i$, respectively, and $N$ denotes the number of Gaussian components involved. The GM distribution's parameters are commonly estimated via the EM [10] method running on the samples of the random variable.

GM distributions are commonly exploited to characterize a large class of sample distributions, primarily due to their ability to form smooth approximations for various densities. In this paper, we study their use in approximating the PDF of the outgoing LLRs from the state nodes under joint decoding. To simplify the calculations, similar to [2], [5], we assume that the LLRs sent from the variable nodes to the state nodes have a Gaussian density. Fig. 2 illustrates the PDF of the outgoing LLRs sent from the state nodes to the variable nodes of component LDPC decoder 1 computed with different methods. $I_{v s}$ denotes the mutual information between the transmitted bits and the LLRs sent from the variable nodes of component LDPC decoder 2 to the state nodes. According to the figure, the PDFs computed via the Monte Carlo simulations match closely with the ones calculated through the analytical derivations. Furthermore, it is clear that the PDFs do not resemble Gaussian densities, instead they are well-approximated with a GM distribution with two Gaussian components. Motivated by these observations, we propose two methods of performance analysis exploiting GM approximation for the LLRs exchanged within the joint decoder. For simplicity of the exposition, the methods are outlined for GM distributions with two components. We note that extensions to a larger number of Gaussian components would follow similar steps.

\section{Proposed Performance Evaluation Methods}

\section{A. Modified DE}

For the proposed method, we utilize the GM approximation and track the PDF of the LLRs exchanged among the nodes of the Tanner graph of the joint decoder. In the following we elaborate on the computations performed for each phase of the decoding iteration separately.

State Node to Variable Node: We exploit the GM assumption to characterize the PDF of the outgoing LLRs from the state nodes. To estimate the parameters of the GM distributions, we utilize the EM method on the samples of the actual PDF computed through (3) based on the samples of the received LLRs from the variable nodes of the other LDPC component decoder. These samples are generated by applying the inverse transform sampling technique [19] on the corresponding PDF obtained in the previous iteration. To reduce the amount of EM computations, the initial estimates of the each parameter of the GM

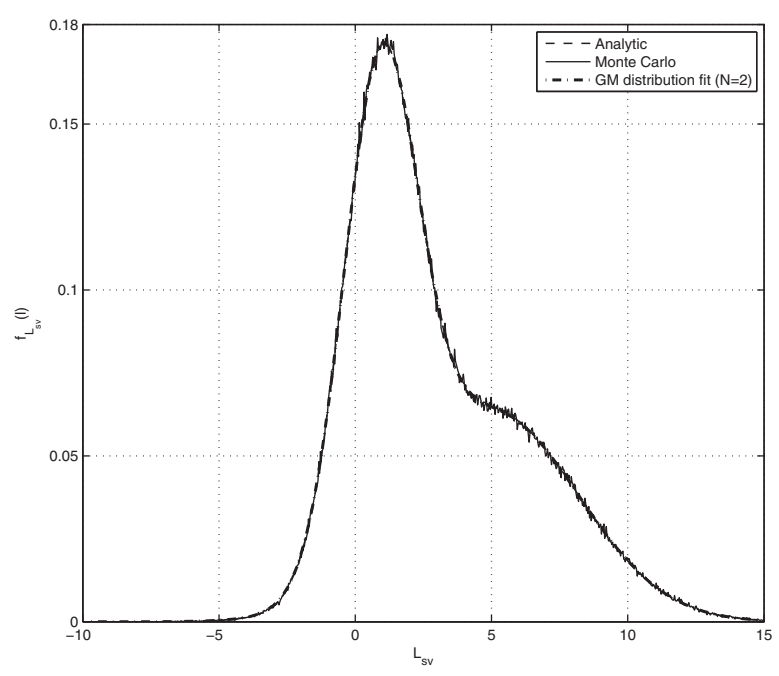

(a) $P_{1}=4 \mathrm{~dB}, P_{2}=4 \mathrm{~dB}, I_{v s}=0.3$

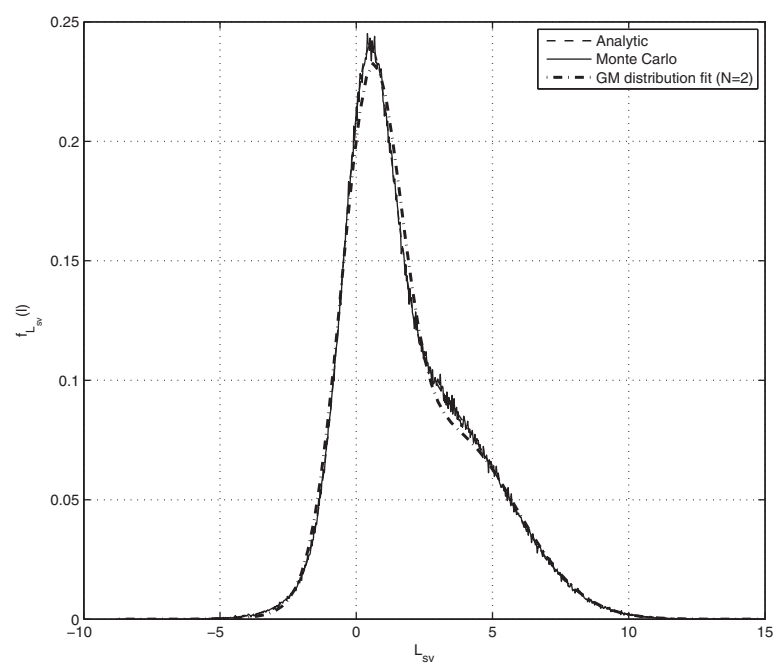

(b) $P_{1}=2 \mathrm{~dB}, P_{2}=1 \mathrm{~dB}, I_{v s}=0.1$.

Fig. 2. Illustration of the PDFs of the outgoing LLRs from the state nodes to the variable nodes of the component LDPC decoder of user 1 for different values $I_{v s}$.

distribution at each iteration can be chosen as the value for the corresponding parameter estimated in the previous iteration.

Variable Node to Check Node: At the variable node with degree $k$, the LLR $L_{v c_{j}}^{(k)}$ sent on the $j$ th edge is computed as $L_{v c_{j}}^{(k)}=L_{s v}+\sum_{\substack{i \neq 1 \\ i \neq j}}^{k} L_{c v_{i}}$, where $L_{s v}$ and $L_{c v_{i}}$ are the LLRs received from the connected stated node and the $i$ th connected check node, respectively. For a cycle free Tanner graph, the incoming messages at each node are i.i.d, hence the PDF of $L_{v c}^{(k)}$ is obtained as $f_{L_{v c}^{(k)}}=f_{L_{s v}} \otimes\left(\otimes_{i=1}^{d_{v}-1} f_{L_{c v}}\right)$ where $\otimes$ denotes the convolution operation. Considering all the variable nodes, the PDF of the $L_{v c}$ is computed as $f_{L_{v c}}=\sum_{i=2}^{d_{v}} \lambda_{i} \cdot f_{L_{v c}^{(k)}}$.

Check Node to Variable Node: At the check node with degree $k$, the LLR $L_{c v_{j}}^{(k)}$ sent on the $j$ th edge is computed as $L_{c v_{j}}^{(k)}=2 \tanh ^{-1}\left(\prod_{\substack{i \neq 1 \\ i \neq j}}^{k} \tanh \left(\frac{L_{v c_{i}}}{2}\right)\right)$. Due to the non-linearity of the update rule, the PDF of $L_{c v}^{(k)}$ is typically computed via a look-up table [11]. In this paper, we follow a similar 
approach where the PDF is calculated by applying a twoinput operator $R$, that is, $f_{L_{c v}^{(k)}}=R^{k-1} f_{L_{v c}}$, where $R(a, b)=$ $\mathcal{Q}\left(2 \tanh ^{-1}\left(\tanh \left(\frac{a}{2}\right) \tanh \left(\frac{b}{2}\right)\right)\right)$ with $\mathcal{Q}($.$) representing the$ quantization operator. Considering all the check nodes, the PDF of $L_{c v}$ is obtained as $f_{L_{c v}}=\sum_{i=2}^{d_{c}} \rho_{i} \cdot f_{L_{c v}^{(k)}}$.

Variable Node to State Node: The outgoing LLR from the variable node with degree $k$ to the connected state node is computed as $L_{s v}=\sum_{i=1}^{k} L_{c v_{i}}$, therefore $f_{L_{v s}^{k}}=\bigotimes_{i=1}^{k} f_{L_{c v}}$ and $f_{L_{v s}}=\sum_{i=2}^{d_{v}} \bar{\lambda}_{i} \cdot f_{L_{v s}^{(k)}}$ where $\bar{\lambda}_{i}$ represents the node degree distribution computed as $\bar{\lambda}_{i}=\frac{\lambda_{i}}{\sum_{j=2}^{d_{v}} \frac{\lambda_{j}}{j}}$.

Yedla et. al. in [7] also utilize the DE method to analyze the performance of the joint decoder employed for the two-user GMAC. They exploit look-up tables to characterize the PDFs of the outgoing LLRs from the state nodes. Despite the accuracy of the method, considerable amount of memory is required to construct the look-up tables. Compared to [7], in our proposed method the PDF of the outgoing LLRs from the state nodes are approximated with a GM rather than being exactly characterized. Moreover, the complexity of our method does not scale with the number of involved component LDPC decoders hence it is more amenable for extension to higher number of users. The proposed approach can also be compared to the full Monte Carlo simulation method adopted in [13], where, due to the a priori GM assumption on the PDF, the proposed method is more efficient hence it requires fewer samples to approximate the PDF.

We observe through examples that for large values of powers, when the channel gains are equal, the PDFs of the outgoing LLRs from the state nodes contain spikes around zero which cannot be well approximated with GM distributions leading to poor decoding threshold estimates.

\section{B. Modified EXIT Analysis}

We exploit the GM approximation in tracking the evolution of the mutual information between the transmitted BPSK symbol $X$ and the exchanged LLR $L$. It is shown in [13] that under joint decoding the symmetry property of the exchanged LLRs is preserved, therefore the associated mutual information can be obtained as [20]

$$
I(X ; L)=1-E\left\{\log _{2}(1+\exp (-L))\right\},
$$

where the expectation is taken over $L$. For $L$ with a GM distribution with $N$ Gaussian components, (8) can be computed as

$$
\begin{gathered}
I(X ; L)=1-\int_{-\infty}^{\infty}\left(\sum_{i=1}^{N} \frac{w_{i}}{\sqrt{2 \pi \sigma_{i}^{2}}} \exp \left(-\frac{\left(l-\mu_{i}\right)^{2}}{2 \sigma_{i}^{2}}\right)\right. \\
\left.\quad \times \log _{2}(1+\exp (-l))\right) d l \\
=\sum_{i=1}^{N} w_{i} J^{\prime}\left(\mu_{i}, \sigma_{i}\right),
\end{gathered}
$$

TABLE I

DeCODING Thresholds OF THE OPTIMIZEd LDPC CODES IN [2] COMPUTED With DifFERENT MEthods of EXIT ANALYSis

\begin{tabular}{|c|c|c|c|c|}
\hline$R$ & $P^{*}$ & $P_{(1)}^{*}$ & $P_{(2)}^{*}$ & $P_{(3)}^{*}$ \\
\hline 0.3 & -1.61 & -1.75 & -1.73 & -1.64 \\
\hline 0.4 & 0.32 & 0.35 & 0.32 & 0.32 \\
\hline 0.5 & 2.19 & 2.11 & 2.11 & 2.19 \\
\hline 0.6 & 4.4 & 4.16 & 4.01 & 4.31 \\
\hline
\end{tabular}

where $N=2$ for the proposed method and $J^{\prime}(\mu, \sigma)$ is defined as

$$
\begin{aligned}
J^{\prime}(\mu, \sigma)= & 1-\frac{1}{\sqrt{2 \pi \sigma^{2}}} \int_{-\infty}^{\infty} \exp \left(-\frac{(l-\mu)^{2}}{2 \sigma^{2}}\right) \\
& \times \log _{2}(1+\exp (-l)) d l
\end{aligned}
$$

The $J^{\prime}$ function is analytically calculated in Appendix A. The introduced function can be considered as an extension to the $J$ function in [12]; however, no specific relation is assumed between the mean and the variance in the computation. In the following, we detail the approach taken to compute the mutual information associated with the exchanged LLRs between the different nodes of the Tanner graph, separately.

State Node to Variable Node: We consider a GM distribution for the PDF of $L_{s v}$. To characterize the associated GM distribution, we generate samples of the outgoing LLRs through (4) based on the samples of the received LLRs from the other component LDPC decoder whose PDF is approximated with $\mathcal{N}\left(\mu_{v s}, 2 \mu_{v s}\right)$ where $\mu_{v s}=\frac{J^{-1}\left(I_{v s}\right)}{2}$. The EM method is then utilized to calculate the parameters of the GM distribution. The mutual information associated with $L_{s v}$ is computed via (9). Note that at the zeroth iteration $\mu_{v s}=I_{v s}=0$.

Variable Node to Check Node: Considering the factor graph, the outgoing LLR sent on an edge from each variable node is computed by adding the received LLRs from the connected check nodes and the neighboring state node. Assuming the factor graph of the joint decoder is cycle free, the Central Limit Theorem (CLT) can be invoked to approximate the PDF of the added LLRs received from the check nodes with a Gaussian density. As a consequence, the PDF of the outgoing LLRs can be computed as the convolution of a Gaussian density with a GM distribution, which results in a GM distribution. The parameters of the GM distribution corresponding to the variable nodes with degree $k$ are computed as

$$
\begin{aligned}
& \mu_{v c_{m}}^{(k)}=(k-1) \mu_{c v}+\mu_{s v_{l}}, \\
& \sigma_{v c_{m}}^{(k)}=\sqrt{(k-1) \sigma_{c v}^{2}+\sigma_{s v_{l}}^{2}}, \\
& w_{v c_{m}}^{(k)}=w_{s v_{m}},
\end{aligned}
$$

where $\mu_{s v_{m}}$ and $\sigma_{s v_{m}}$ denote the mean value and the standard deviation of the $m$ th $(m=1,2)$ Gaussian component of the GM distribution associated with $L_{s v}$, respectively. The computed GM distribution parameters in (11) can then be used 

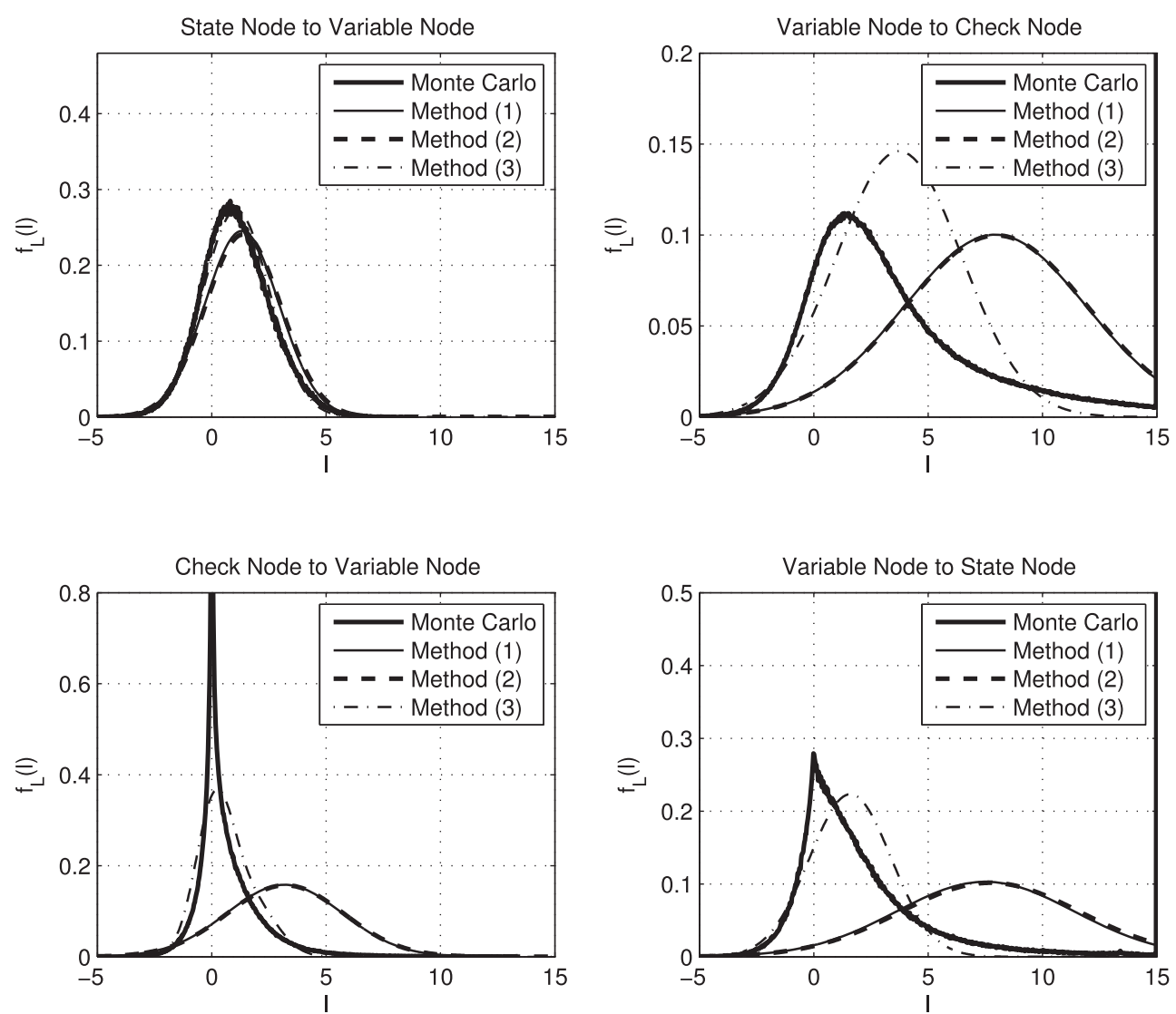

Fig. 3. Comparison of the different methods in characterizing the PDFs of the LLRs exchanged in the joint decoder at iteration 100 for the optimized code in [2] corresponding to the code rate $R=0.3$.

towards computation of the associated mutual information $I_{v c}$ calculated as

$$
\begin{aligned}
I_{v c}\left(X ; L_{v c}\right)=\sum_{i=2}^{d v} \lambda_{i} \cdot( & w_{v c_{1}}^{(i)} J^{\prime}\left(\mu_{v c_{1}}^{(i)}, \sigma_{v c_{1}}^{(i)}\right) \\
& \left.+w_{v c_{2}}^{(i)} J^{\prime}\left(\mu_{v c_{2}}^{(i)}, \sigma_{v c_{2}}^{(i)}\right)\right) .
\end{aligned}
$$

Check Node to Variable Node: $L_{c v}$ is a non-linear function of $L_{v c}$, therefore we approximate the PDF of $L_{c v}$ with a GM distribution computed based on the samples of $L_{v c}$. For ease of computation, the samples are drawn from $\mathcal{N}\left(\mu_{v c}, 2 \mu_{v c}\right)$ where $\mu_{v c}=\frac{\left(J^{-1}\left(I_{v c}\right)\right)^{2}}{2}$ with $J^{-1}$ introduced in [12]. The mutual information associated with $L_{c v}$ is obtained similar to (12).

Variable Node to State Node: The computation of $L_{v s}$ is performed by simply adding the received LLRs from the connected check nodes. Assuming the factor graph of the joint decoder is cycle-free, the CLT can be involved approximating the PDF of $L_{v s}^{(k)}$ with a Gaussian density with $\mu_{v s}^{(k)}=k \cdot \bar{\mu}_{c v}$ and $\sigma_{v s}^{(k)}=\sqrt{k} \cdot \bar{\sigma}_{c v}$ where $\bar{\mu}_{c v}$ and $\bar{\sigma}_{c v}$ denote the mean and variance of the LLRs received from the check nodes, respectively. We have

$$
\begin{aligned}
& \bar{\mu}_{c v}=w_{c v_{1}} \mu_{c v_{1}}+w_{c v_{2}} \mu_{c v_{2}}, \\
& \bar{\sigma}_{c v}=\sqrt{w_{c v_{1}}\left(\mu_{c v_{1}}^{2}+\sigma_{c v_{1}}^{2}\right)+w_{c v_{2}}\left(\mu_{c v_{2}}^{2}+\sigma_{c v_{2}}^{2}\right)-\left(\bar{\mu}_{c v}\right)^{2}} .
\end{aligned}
$$

The average mutual information associated with $L_{v s}$ is computed as $I_{v s}\left(X ; L_{v s}\right)=\sum_{i=2}^{d_{v}} \bar{\lambda}_{i} \cdot J^{\prime}\left(\mu_{v s}^{(i)}, \sigma_{v s}^{(i)}\right)$.
To assess the performance, we compute the decoding thresholds for the optimized degree distributions in [2] utilizing the proposed and the existing methods of EXIT analysis. Table I shows the decoding thresholds computed in terms of the average received power measured in $\mathrm{dB}$. We refer to the methods of [2], [5], and the modified EXIT analysis as method (1), method (2), and method (3), respectively. $P^{*}$ denotes the true decoding threshold estimates obtained with the Monte Carlo simulations. $P_{(1)}^{*}, P_{(2)}^{*}$, and $P_{(3)}^{*}$ represent the values of the decoding thresholds computed via the methods (1), (2), and (3), respectively. According to the table, our proposed method provides better estimates of the decoding thresholds compared to the two other methods. This superiority is especially prominent for the case of $R=0.6$. Fig. 3 demonstrates the PDF of the LLRs corresponding to the optimized degree distributions in [2] for a two-user GMAC with equal channel gains computed via different methods. It can be observed that our proposed method provides more accurate PDF estimates compared to the methods adopted in [2] and [5]. According to Fig. 3, the GM approximation matches with the PDF of the outgoing LLRs from the state nodes; however, such accuracy is not achieved for the PDF associated with the check nodes.

\section{LDPC CODE OPTIMIZATION}

In order to design ensemble of good LDPC codes for GMACs, we utilize an instance of differential evolution [21]. The optimization process is initialized with two LDPC code 
TABLE II

Optimized Degree Distributions for Equal Channel Gains Scenario

\begin{tabular}{|c|c|c|c|c|c|c|c|c|c|c|c|c|c|}
\hline$R$ & $d_{c}$ & $P^{*}(\mathrm{~dB})$ & $\lambda_{2}$ & $\lambda_{3}$ & $\lambda_{4}$ & $\lambda_{5}$ & $\lambda_{6}$ & $\lambda_{7}$ & $\lambda_{8}$ & $\lambda_{13}$ & $\lambda_{20}$ & $\lambda_{21}$ & $\lambda_{100}$ \\
\hline 0.3 & 6 & -1.73 & 0.2741 & 0.2113 & 0.0078 & 0.0178 & 0.0206 & 0.0063 & 0.0239 & 0.1992 & 0 & 0 & 0.2389 \\
\hline 0.6 & 9 & 4.25 & 0.4771 & 0.0744 & 0 & 0 & 0 & 0 & 0 & 0 & 0.1322 & 0.1231 & 0.1931 \\
\hline
\end{tabular}

ensembles selected from the P2P codes optimized for the BIAWGN channel utilizing the method of EXIT analysis in [12]. The adopted codes for a GMAC with fixed channel gains are referred to as admissible if they lead to asymptotically error free decoding. For the case of quasi-static fading scenario, the employed degree distribution are called admissible if they asymptotically lead to error-free decoding for $1-P_{o}$ of the considered channel realizations computed through Monte Carlo simulations.

The admissibility of the employed degree distributions can be verified through tracking the evolution of the PDF or the mutual information associated with the LLRs exchanged within the joint decoder. For the next step of the code optimization, the obtained admissible degree distributions are modified via the perturbing vectors. For the general case, both variable node and check node degree distributions are perturbed, however, to simplify the optimization, we consider a singleton distribution for the check node degrees, therefore only the variable node degree distribution is perturbed.

The $i$ th polynomial coefficient of variable node degree distribution is perturbed as $\tilde{\lambda}_{i}=\lambda_{i}+e_{i}$, where $e_{i}$ is the $i$ th element of the perturbing vector e. For simplification, we only perturb the non-zero values of the polynomial coefficient of the initial degree distributions, i.e., $e_{i}=0$ if $\lambda_{i}=0$. The perturbed degree distribution should satisfy $\tilde{\lambda}(1)=1,0 \leq \tilde{\lambda}_{i} \leq 1$ implying

$$
\sum_{i=2}^{d_{v}} e_{i}=0,0 \leq \lambda_{i}+e_{i} \leq 1
$$

To control the variations at each iteration, it is beneficial to limit the variance of the elements of the perturbing vector $\sigma_{e}^{2}$ computed as

$$
\sigma_{e}^{2}=\sum_{i=2}^{d_{v}} e_{i}^{2}
$$

LDPC codes can be optimized with different objectives such as rate maximization or SNRs minimization. For rate maximization the decoding threshold is fixed, and at each iteration of the perturbation, the code rates of the employed degree distributions are incremented, therefore the perturbing vector should satisfy $1-\frac{1}{d_{c}} \frac{1}{\sum_{i=2}^{d_{v}} \frac{\tilde{\lambda}_{i}}{i}}=r_{0}+\Delta$, where $\Delta$ denotes the rate increment. This constraint can be written as $\sum_{i=2}^{d_{v}} \frac{\tilde{\lambda}_{i}}{i}=$ $\frac{1}{d_{c}} \frac{1}{1-\left(r_{0}+\Delta\right)}$, which is equivalent to

$$
\sum_{i=2}^{d_{v}} \frac{e_{i}}{i}=\frac{\Delta / d_{c}}{\left(1-r_{0}\right)^{2}-\Delta\left(1-r_{0}\right)} .
$$

For minimization of required SNRs, the code rates are kept fixed, hence (15) is simplified to $\sum_{i=2}^{d_{v}} \frac{e_{i}}{i}=0$. At each iteration of the code optimization, the admissibility of the degree distributions are checked for a decrease in the received powers.

To generate the perturbing vector, we can draw all the elements except three from a normal distribution, i.e., $\mathcal{N}(0,1)$, and compute the remaining elements by solving (13), (14) and (15). The perturbing vector is adopted if it satisfies the inequality constraint (13) and the stability condition (2), otherwise a new perturbing vector is generated. The perturbed degree distributions will replace the initial degree distributions if they are admissible, otherwise they are dismissed and a new iteration is performed. The code optimization is concluded if new admissible degree distributions cannot be found after a predetermined number of iterations. Therefore, the last pair of admissible degree distributions is the optimum.

\section{NumERICAL EXAMPLES}

\section{A. Fixed Channel Gains}

For this scenario, we incorporate the proposed modified methods of EXIT analysis and DE into the LDPC code optimization with the objective of minimization of required SNRs. For equal channel gains, we compare our designed codes with those corresponding to the code rates 0.3 and 0.6 in [2]. We employ the designed degree distributions in [2] to initialize the code optimization. We utilize the modified DE to design codes for the code rate 0.3 . For the code rate 0.6 , we perform the code optimization employing the modified EXIT analysis.

Table II presents the resulting optimized degree distributions whose decoding thresholds are denoted by $P^{*}$ computed via Monte Carlo simulation. The decoding thresholds of the optimized degree distributions [2] associated with code rates 0.3 and 0.6 are $-1.61 \mathrm{~dB}$ and $4.4 \mathrm{~dB}$ which are inferior to our optimized codes by $0.12 \mathrm{~dB}$ and $0.15 \mathrm{~dB}$, respectively.

We also compute the performance of the P2P codes optimized for the BI-AWGN channel when employed over the two-user GMAC. The degree distributions are optimized for the code rates associated with those designed in [2]. The decoding thresholds of the degree distributions corresponding to code rates $0.3,0.4,0.5$ are $-0.81 \mathrm{~dB}, 1.96 \mathrm{~dB}$, and $5.06 \mathrm{~dB}$. For the case of code rate 0.6 , the $\mathrm{P} 2 \mathrm{P}$ codes are not supported over the two-user GMAC even if there is no noise. These findings suggest that optimized codes achieve considerable improvement over P2P when employed for the two-user GMAC with equal channel gains.

For unequal channel gains, there are no specific designs in the literature. So, we consider a degree profile with maximum degree 50 and choose the non-zero variable node degrees as 2 , $3,4,9,10,19,20,49,50$. Although there is no guarantee that this is the best choice, the selected degree profile is motivated by the pattern suggested in [6] for the optimized codes over the BI-AWGN channel for which the non-zero variable node 
TABLE III

Optimized Degree Distributions for Unequal Channel Gains Scenario $\left(R_{1}=0.486, R_{2}=0.059\right)$

\begin{tabular}{|c|c|c|c|c|c|c|c|c|c|c|c|c|}
\hline \multirow[b]{3}{*}{ lified E2 } & $P_{1}^{*}(\mathrm{~d}$ & Isg. & $d_{c}$ & $\lambda_{2}$ & $\lambda_{3}$ & $\lambda_{4}$ & $\lambda_{9}$ & $\lambda_{10}$ & $\lambda_{19}$ & $\lambda_{20}$ & $\lambda_{49}$ & $\lambda_{50}$ \\
\hline & \multirow{2}{*}{22} & $X_{1}$ & 8 & 0.2023 & 0.2635 & 0.0770 & 0.1730 & 0.0654 & 0.0948 & 0.0557 & 0.0547 & 0.01 \\
\hline & & $X_{2}$ & 3 & & & & 0.0 & & & & & 0.1 \\
\hline & & $X_{1}$ & 8 & & & 0. & & & & & & \\
\hline & & $X_{2}$ & 3 & & & & & & & & & 0.02 \\
\hline & & $X_{1}$ & 8 & & & 0.1 & $0.0^{7}$ & & & & & 0.06 \\
\hline & & $X_{2}$ & 3 & & $25 / 8$ & & 0.1214 & & & & & .0 \\
\hline & & $X_{1}$ & 8 & 145 & & & $10+2$ & & & & & \\
\hline & & $\sqrt{\bar{y}}$ & 3 & 0.4770 & .2569 & 0.0597 & 0.0579 & 0.0402 & 92 & .0433 & 0210 & \\
\hline
\end{tabular}

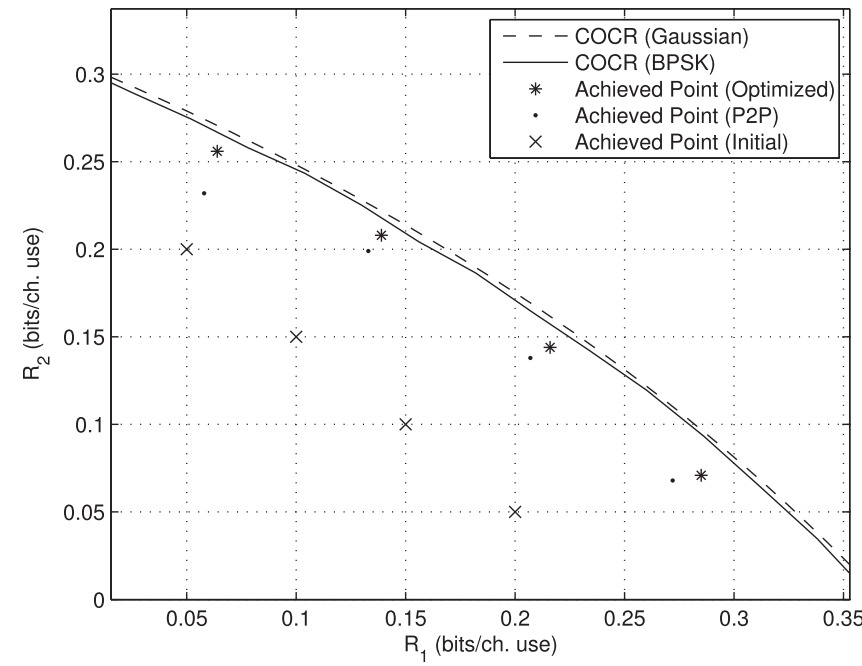

Fig. 4. COCRs of Gaussian and BPSK signaling along with the optimized and the $\mathrm{P} 2 \mathrm{P}$ codes. $P_{1}=5 \mathrm{~dB}, P_{2}=4 \mathrm{~dB}$, and $P_{\mathrm{o}}=0.1$.

degrees are distributed around the minimum and maximum degrees and a few values in between. Similar pattern is also followed by codes designed in [2], [5].

We consider $P_{1}=0 \mathrm{~dB}$ and $P_{2}=-8 \mathrm{~dB}$ and select the rate pair $(0.486,0.059)$, which corresponds to a corner point of the dominant face of the capacity region, as the rates of the employed LDPC codes. For code optimization, we select a pair of admissible off-the-shelf P2P codes with similar code rates as the initial degree distributions. During the code optimization process, we start with higher values for the received powers and decrease the values at each iteration keeping the power ratio unchanged, i.e., $\frac{P_{1}}{P_{2}}=6.31$ throughout the code optimization. We perform separate code designs utilizing the proposed modified EXIT analysis and DE method. The decoding thresholds of the optimized codes are compared against the ones obtained via the method (2). Furthermore, we calculate the decoding threshold of the off-the-shelf P2P codes optimized over BI-AWGN channels when they are employed for the GMAC with the constraint $\frac{P_{1}}{P_{2}}=6.31$. Table III demonstrates the degree distributions for the optimized codes. The decoding thresholds are computed via Monte Carlo simulations and are provided in terms of $P_{1}^{*}$ with $P_{2}^{*}=\frac{P_{1}^{*}}{6.31}$. It is clear from the table that the codes designed via the proposed methods outperform the ones optimized with method (2) and the P2P codes.

\section{B. Quasi-Static Fading}

To illustrate the code design principles for the fading case, we provide several examples. Two scenarios of real and complex channel gains are considered. We declare a pair of degree distributions admissible if the computed decoding behavior, measured in frame error rate (FER), meets the given outage probability asymptotically. The accuracy of the computations relies on the number of channel realizations taken into account. In our designs, we consider the outage probability of 0.1 and perform the computations for $10^{4}$ channel realizations. It is easy to check that for the considered number of channel realizations the associated outage probability is bounded as $0.0941<P_{o}<$ 0.1059 for a $95 \%$ confidence level.

Due to extensive computations, for the case of quasi-static fading the proposed methods of decoding threshold estimation (the modified DE and the modified EXIT analysis) is not efficient in the current form to be incorporated into the code optimization. Hence, we employ the EXIT chart analysis in [5] wherein the evolution of the mutual information is computed through a simple linear approximation and LLRs are assumed to have Gaussian distribution. Note that the linear approximation used in [5] does not result in accurate decoding thresholds for some ranges of the power values; however, the simplicity of the method renders it very efficient for the involved computations under the quasi-static fading scenario.

Fig. 4 illustrates the COCRs for Gaussian and BPSK signaling computed for real channel gains. Code optimization is performed for four instances of rate pairs with the goal of rate maximization. The initial degree distributions are picked from the P2P codes designed for the BI-AWGN channel. For each instance, the trajectory of the rate increments is a straight line passing through the origin. Table IV shows the degree distributions of the optimized codes and those of the available P2P codes. Fig. 4 presents the achieved rate pairs employing the optimized codes and the best P2P ones clearly demonstrating the superiority of the newly optimized codes. Finally, Fig. 5 shows the FERs for finite block lengths of the specific codes selected from the optimized degree distributions corresponding to the code rate pair $(0.139,0.208)$ where the FERs associated with $1 \mathrm{k}$ and $10 \mathrm{k}$ are $1.75 \mathrm{~dB}$ and $1.1 \mathrm{~dB}$ away from the outage limit, respectively at an FER of 0.1 . The newly designed codes provide better performance than the $\mathrm{P} 2 \mathrm{P}$ codes for the rate pair $(0.133,0.199)$ (at an FER of 0.1) as well.

As the second example, we consider a quasi-static fading channel with complex channel gains. Since characterization of the COCR for the BPSK signaling is difficult, we calculate 
TABLE IV

Optimized Degree Distributions (Real Channel Gains), $P_{1}=5 \mathrm{~dB}, P_{2}=4 \mathrm{~dB}, P_{o}=0.1$

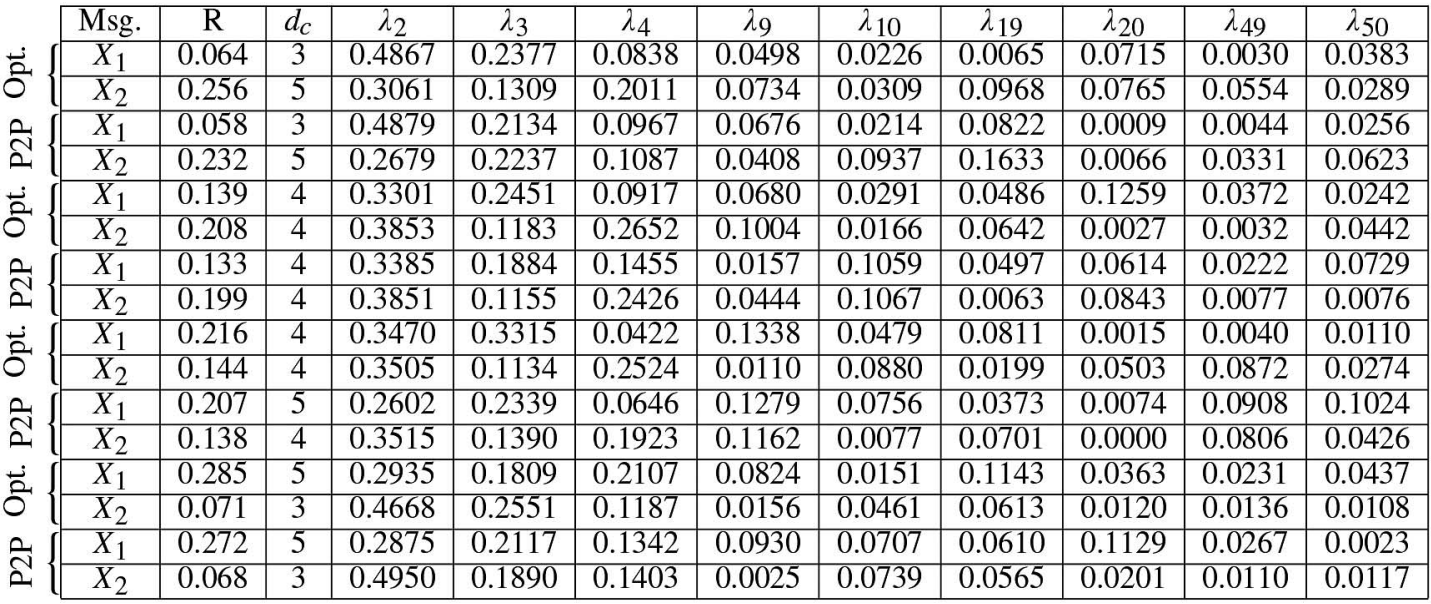

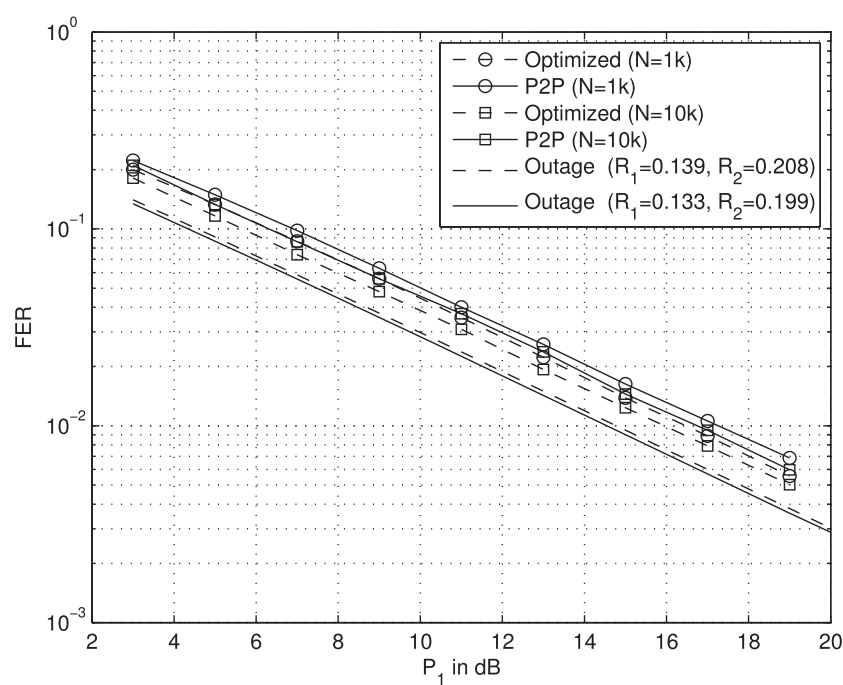

Fig. 5. FER of the optimized codes and the P2P codes employing real channel gains.

the Gaussian signaling COCR as an outer bound. Similar to the previous example, we perform the code optimization for four instances and compare them with the P2P codes optimized for the BI-AWGN channel. Degree distributions are shown in Table V for the optimized codes and the reference P2P ones. Achieved rate pairs are shown in Fig. 6. Fig. 7 demonstrates the decoding results for finite block length codes picked from the optimized degree distributions corresponding to the code rate pair $(0.289,0.072)$. At an FER of 0.1 , the code block lengths with $1 \mathrm{k}$ and $10 \mathrm{k}$ operate $1.6 \mathrm{~dB}$ and $1.25 \mathrm{~dB}$ away from the outage limit computed for Gaussian signaling, respectively. Furthermore, the optimized codes offer better performance than the P2P codes corresponding to the rate pair $(0.268,0.067)$ as well.

\section{CONCLUSIONS}

We studied the problem of LDPC code design for the twouser GMAC exploring two scenarios of fixed and quasi-static fading channel gains. Considering joint decoding of the two users coded bits, we characterized the PDF of the outgoing LLRs from the state nodes and observed that it can be well approximated with GM distributions. We then exploited the GM approximation to develop variants of existing DE and EXIT analysis methods. We utilized the newly proposed methods to design codes for fixed channel gain scenarios and showed that the optimized codes obtained via the proposed methods offer better performance than the P2P codes and those achieved from the already existing. For the quasi-static fading case, we adopted an existing (simple) implementation of EXIT analysis and performed code optimization for real and complex channel gains. The optimized codes improve upon the $\mathrm{P} 2 \mathrm{P}$ codes in this case too. Finally, simulations with finite length codes picked from the designed ensembles demonstrate that the performance of the optimized codes is close to the outage limits.

\section{APPENDIX}

In this appendix, we compute the defined function $J^{\prime}(\mu, \sigma)$ in (10), given by

$$
\begin{aligned}
J^{\prime}(\mu, \sigma)= & 1-\frac{1}{\sqrt{2 \pi \sigma^{2}}} \int_{-\infty}^{\infty} \exp \left(-\frac{(z-\mu)^{2}}{2 \sigma^{2}}\right) \\
& \times \log _{2}(1+\exp (-z)) d z .
\end{aligned}
$$

For the ease of exposition, we write $J^{\prime}(\mu, \sigma)$ as $J^{\prime}(\mu, \sigma)=$ $1-\frac{I}{\log (2) \sqrt{2 \pi \sigma^{2}}}$. Also, $I$ can be split into two parts $I_{1}$ and $I_{2}$; that is $I=I_{1}+I_{2}$, where

$$
\begin{aligned}
& I_{1}=\int_{-\infty}^{0} \exp \left(-\frac{(z-\mu)^{2}}{2 \sigma^{2}}\right)(\log (1+\exp (z))-z) d z, \\
& I_{2}=\int_{0}^{\infty} \exp \left(-\frac{(z-\mu)^{2}}{2 \sigma^{2}}\right) \log (1+\exp (-z)) d z .
\end{aligned}
$$

$I_{1}$ can be expressed as $I_{1}=I_{11}-I_{12}$ where

$$
\begin{aligned}
I_{12} & =\int_{-\infty}^{0} z \exp \left(-\frac{(z-\mu)^{2}}{2 \sigma^{2}}\right) d z \\
& =\int_{-\infty}^{-\frac{\mu}{\sigma}}(\sigma t+\mu) \exp \left(-\frac{t^{2}}{2}\right) \sigma d t
\end{aligned}
$$


TABLE V

Optimized Degree Distributions (Complex Channel Gains), $P_{1}=5 \mathrm{~dB}, P_{2}=4 \mathrm{~dB}, P_{o}=0.1$

\begin{tabular}{|c|c|c|c|c|c|c|c|c|c|c|c|c|}
\hline \multirow{3}{*}{$\vec{\approx}$} & Msg. & $\mathrm{R}$ & $d_{c}$ & $\lambda_{2}$ & $\lambda_{3}$ & $\lambda_{4}$ & $\lambda_{9}$ & $\lambda_{10}$ & $\lambda_{19}$ & $\lambda_{2}$ & $\lambda_{4}$ & $\lambda_{5}$ \\
\hline & $X_{1}$ & 0.068 & 3 & 0.4906 & 0.3029 & 0.0021 & 0.0125 & 0.0254 & 0.0963 & 0.0117 & 0.0243 & \\
\hline & $X_{2}$ & 0.272 & 5 & 0.2593 & 0.2876 & 0.1044 & 0.0948 & 0.0445 & 0.0599 & 0.0673 & 0.0252 & 0.0570 \\
\hline & $X_{1}$ & 0.059 & 3 & 0.4770 & 0.2569 & 0.0597 & 0.0579 & 0.0402 & 0.0392 & 0.0433 & 0.0210 & 0.0049 \\
\hline & $X_{2}$ & 0.234 & 5 & 0.2790 & 0.1898 & 0.1271 & 0.0680 & 0.1133 & 0.0895 & 0.0093 & 0.0838 & 0.0403 \\
\hline & $X_{1}$ & 0.135 & 4 & 0.3344 & 0.2055 & 0.1097 & 0.1235 & 0.0597 & 0.0461 & 0.0409 & 0.0415 & 0.0387 \\
\hline & $X_{2}$ & 0.202 & 4 & 0.3732 & 0.2722 & 0.0354 & 0.1051 & 0.1222 & 0.0199 & 0.0250 & 0.0167 & 0.0304 \\
\hline & $\overline{X_{1}}$ & 0.12 & 4 & 0.3243 & 0.2267 & 0.0965 & 0.0850 & 0.0743 & 0.0233 & 0.0248 & 0.0704 & 0.0748 \\
\hline & $X_{2}$ & 0.18 & 4 & 0.3587 & 0.2273 & 0.0973 & 0.1078 & 0.0848 & 0.0193 & 0.0613 & 0.0228 & 0.0206 \\
\hline & $X_{1}$ & 0.21 & 4 & 0.3958 & 0.2366 & 0.0903 & 0.0747 & 0.0121 & 0.0117 & 0.1122 & 0.0576 & 0.0090 \\
\hline & $X_{2}$ & 0.14 & 4 & 0.3254 & 0.1985 & 0.1763 & 0.0740 & 0.0072 & 0.0255 & 0.1201 & 0.0429 & 0.0300 \\
\hline & $X_{1}$ & 0.176 & 4 & 0.3554 & 0.2131 & 0.1220 & 0.1638 & 0.0028 & 0.0787 & 0.0078 & 0.0363 & 0.0200 \\
\hline & $X_{2}$ & 0.117 & 4 & 0.3438 & 0.1145 & 0.2342 & 0.0087 & 0.0426 & 0.0474 & 0.0859 & 0.0345 & 0.0883 \\
\hline & $X_{1}$ & 0.289 & 5 & 0.2634 & 0.3123 & 0.0900 & 0.0887 & 0.0448 & 0.0697 & 0.0779 & 0.0326 & 0.0205 \\
\hline & $X_{2}$ & 0.072 & 3 & 0.5259 & 0.1626 & 0.0962 & 0.0643 & 0.0823 & 0.0133 & 0.0258 & 0.0012 & 0.0283 \\
\hline & $X_{1}$ & 0.268 & 5 & 0.2948 & 0.2026 & 0.1153 & 0.1107 & 0.0959 & 0.0188 & 0.1104 & 0.0399 & 0.0116 \\
\hline & $X_{2}$ & 0.067 & 3 & 0.4910 & 0.2239 & 0.0849 & 0.1004 & 0.0002 & 0.0320 & 0.0578 & 0.0059 & 0.0040 \\
\hline
\end{tabular}

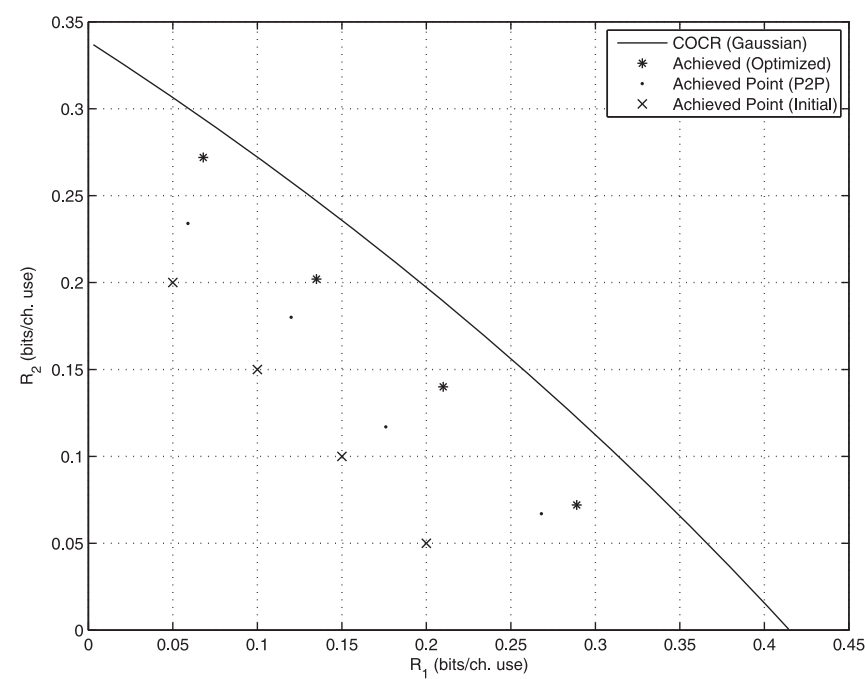

Fig. 6. COCR for the Gaussian signaling and the achieved rate pairs. $P_{1}=5$ $\mathrm{dB}, P_{2}=4 \mathrm{~dB}, P_{\mathrm{o}}=0.1$.

$$
\begin{aligned}
& =\left(\int_{-\infty}^{-\frac{\mu}{\sigma}} \sigma^{2} t \exp \left(-\frac{t^{2}}{2}\right) d t+\mu \sigma \int_{-\infty}^{-\frac{\mu}{\sigma}} \exp \left(-\frac{t^{2}}{2}\right) d t\right) \\
& =\left(-\sigma^{2} \exp \left(-\frac{\mu^{2}}{2 \sigma^{2}}\right)+\mu \sigma \sqrt{\frac{\pi}{2}}\left(1+\operatorname{erf}\left(-\frac{\mu}{\sqrt{2} \sigma}\right)\right)\right) .
\end{aligned}
$$

Expanding $\log (1+\exp (z))$ to its Taylor series, $I_{11}$ can be rewritten as

$$
\begin{aligned}
I_{11} & =\int_{-\infty}^{0} \exp \left(-\frac{(z-\mu)^{2}}{2 \sigma^{2}}\right)\left(\sum_{m=1}^{\infty}(-1)^{m-1} \frac{\exp (m z)}{m}\right) d z \\
& =\sum_{m=1}^{\infty} \frac{(-1)^{m-1}}{m}\left(\int_{-\infty}^{0} \exp \left(-\frac{(z-\mu)^{2}}{2 \sigma^{2}}\right) \exp (m z) d z\right) .
\end{aligned}
$$

The $m$ th term of the summation in (16), denoted as $A_{m}$, can be computed as

$A_{m}=\int_{-\infty}^{0} \exp \left(-\frac{(z-\mu)^{2}}{2 \sigma^{2}}\right) \exp (m z) d z$

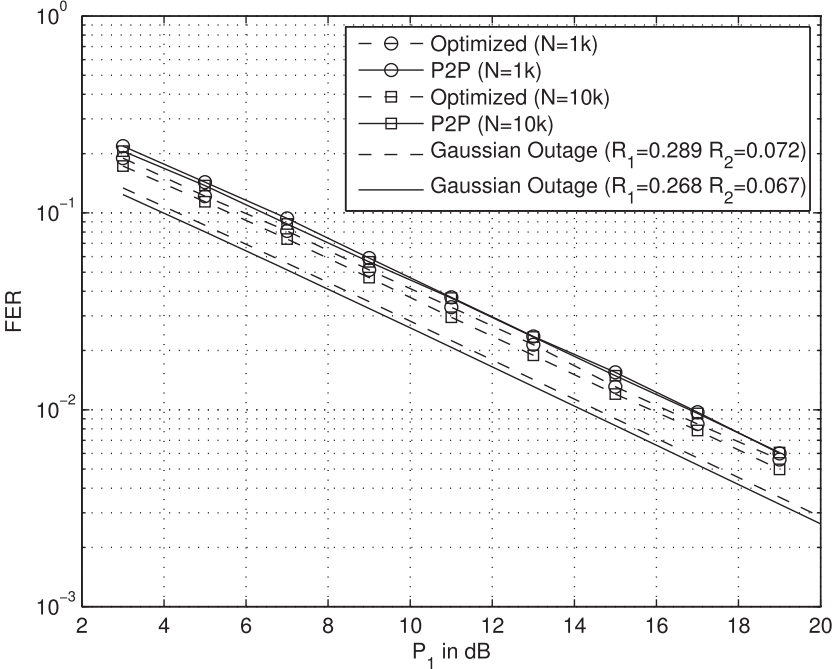

Fig. 7. FER of the optimized codes and the P2P codes employing complex channel gains.

$$
\begin{aligned}
& =\int_{-\infty}^{0} \exp \left(-\frac{z^{2}-2 \mu z+\mu^{2}-2 \sigma^{2} m z}{2 \sigma^{2}}\right) d z \\
& =\int_{-\infty}^{0} \exp \left(-\frac{\left(z-\left(\mu+\sigma^{2} m\right)\right)^{2}-\sigma^{4} m^{2}-2 \sigma^{2} m \mu}{2 \sigma^{2}}\right) d z \\
& =\exp \left(\frac{\sigma^{2} m^{2}}{2}+m \mu\right) \\
& \quad \times\left(\int_{-\infty}^{0} \exp \left(-\frac{\left(z-\left(\mu+\sigma^{2} m\right)\right)^{2}}{2 \sigma^{2}}\right) d z\right)
\end{aligned}
$$

Hence, $I_{11}$ is computed as

$$
\begin{aligned}
I_{11}=\left(\sqrt{2 \pi \sigma^{2}}\right) & {\left[\sum_{m=1}^{\infty} \frac{(-1)^{(m-1)}\left(1-Q\left(-\frac{\mu+m \sigma^{2}}{\sigma}\right)\right)}{m}\right.} \\
& \left.\times \exp \left(\frac{\sigma^{2} m^{2}}{2}+m \mu\right)\right],
\end{aligned}
$$


where $Q(x)=\frac{1}{\sqrt{2 \pi}} \int_{x}^{\infty} \exp \left(-\frac{t^{2}}{2}\right) d t$. Similarly, $I_{2}$ can be written as

$$
\begin{aligned}
I_{2} & =\int_{0}^{\infty} \exp \left(-\frac{(z-\mu)^{2}}{2 \sigma^{2}}\right)\left(\sum_{m=1}^{\infty}(-1)^{m-1} \frac{\exp (-m z)}{m}\right) d z \\
& =\sum_{m=1}^{\infty} \frac{(-1)^{m-1}}{m}\left(\int_{0}^{\infty} \exp \left(-\frac{(z-\mu)^{2}}{2 \sigma^{2}}\right) \exp (-m z) d z\right) .
\end{aligned}
$$

Considering (17), $I_{2}$ is simplified to simplified to

$$
\begin{aligned}
I_{2}=\left(\sqrt{2 \pi \sigma^{2}}\right)\left[\sum_{m=1}^{\infty} \frac{(-1)^{(m-1)} Q\left(-\frac{\mu-m \sigma^{2}}{\sigma}\right)}{m}\right. \\
\left.\quad \times \exp \left(\frac{\sigma^{2} m^{2}}{2}-m \mu\right)\right] .
\end{aligned}
$$

Note that for $\mu=\frac{\sigma^{2}}{2}$, the $J^{\prime}$ reduces to the $J$ function introduced in [12].

\section{REFERENCES}

[1] A. Amraoui, S. Dusad, and R. Urbanke, "Achieving general points in the 2-user Gaussian MAC without time-sharing or rate-splitting by means of iterative coding," in Proc. IEEE Int. Symp. Inf. Theory, Lausanne, Switzerland, Jul. 2002, p. 334

[2] A. Roumy and D. Declercq, "Characterization and optimization of LDPC codes for the 2-user Gaussian multiple access channel," EURASIP J. Wireless Commun. Netw., May 2007, Art. ID. 074890.

[3] R. G. Gallager, "Low-density parity check codes," IRE Trans. Inf. Theory, pp. 21-28, Jan. 1962.

[4] D. J. C. MacKay, "Good error-correcting codes based on very sparse matrices," IEEE Trans. Inf. Theory, vol. 45, no. 2, pp. 399-431, Mar. 1999.

[5] P. Berlin and D. Tuninetti, "LDPC codes for fading Gaussian broadcast channels," IEEE Trans. Inf. Theory, vol. 51, no. 6, pp. 2173-2182, Jun. 2005.

[6] T. Richardson, M. Shokrollahi, and R. Urbanke, "Design of capacityapproaching irregular low-density parity-check codes," IEEE Trans. Inf. Theory, vol. 47, no. 2, pp. 619-637, Feb. 2001.

[7] A. Yedla, P. Nguyen, H. Pfister, and K. Narayanan, "Universal codes for the Gaussian MAC via spatial coupling," in Proc. Commun. Control Comput. (Allerton), Monticello, IL, USA, Sep. 2011, pp. 1801-1808.

[8] S. ten Brink, "Convergence behavior of iteratively decoded parallel concatenated codes," IEEE Trans. Commun., vol. 49, no. 10, pp. 1727-1737, Oct. 2001.

[9] I. Shahid and P. Yahampath, "Distributed joint source-channel code design for GMAC using irregular LDPC codes," EURASIP J. Wireless Commun. Netw., Jan. 2014, Art. ID. 2014:3.

[10] T. K. Moon and W. C. Stirling, Mathematical Methods and Algorithms for Signal Processing. Upper Saddle River, NJ, USA: Prentice-Hall, 2000.

[11] S.-Y. Chung, G. D. Forney Jr., T. Richardson, and R. Urbanke, "On the design of low-density parity-check codes within $0.0045 \mathrm{~dB}$ of the Shannon limit," IEEE Commun. Lett., vol. 5, no. 2, pp. 58-60, Feb. 2001.

[12] S. ten Brink, G. Kramer, and A. Ashikhmin, "Design of low-density parity-check codes for modulation and detection," IEEE Trans. Commun., vol. 52, no. 4, pp. 670-678, Apr. 2004.

[13] S. Sharifi, A. K. Tanc, and T. M. Duman, "Implementing the HanKobayashi scheme using low density parity check codes over Gaussian interference channels," IEEE Trans. Commun., vol. 63, no. 2, pp. $337-$ 350, Feb. 2015.

[14] L. Li, N. Jindal, and A. Goldsmith, "Outage capacities and optimal power allocation for fading multiple-access channels," IEEE Trans. Inf. Theory, vol. 51, no. 4, pp. 1326-1347, Apr. 2005.

[15] A. El Gamal and Y.-H. Kim, Network Information Theory. Cambridge, U.K.: Cambridge Univ. Press, 2011.

[16] R. Narasimhan, "Individual outage rate regions for fading multiple access channels," in Proc. IEEE Int. Symp. Inf. Theory (ISIT), Nice, France, Jun. 2007, pp. 1571-1575.
[17] J. Hou, P. Siegel, L. Milstein, and H. Pfister, "Capacity-approaching bandwidth-efficient coded modulation schemes based on low-density parity-check codes," IEEE Trans. Inf. Theory, vol. 49, no. 9, pp. 21412155, Sep. 2003.

[18] J. Hu and T. M. Duman, "Low density parity check codes over wireless relay channels," IEEE Trans. Wireless Commun., vol. 6, no. 9, pp. 33843394, Sep. 2007.

[19] X. Han and G. R. Liu, Computational Inverse Techniques in Nondestructive Evaluation. Boca Raton, FL, USA: CRC Press, 2003.

[20] J. Hagenauer, "The EXIT chart-Introduction to extrinsic information transfer in iterative processing," in Proc. 12th Eur. Signal Process. Conf. (EUSIPCO), Vienna, Austria, Sep. 2004, pp. 1541-1548.

[21] M. Franceschini, G. Ferrari, R. Raheli, and A. Curtoni, "Serial concatenation of LDPC codes and differential modulations," IEEE J. Sel. Areas Commun., vol. 23, no. 9, pp. 1758-1768, Sep. 2005.

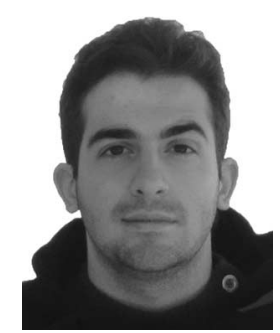

Shahrouz Sharifi (S'10) received the B.S. degree from the University of Tehran, Tehran, Iran, the M.S. degree from Sharif University of Technology, Tehran, Iran, and the Ph.D. degree from Arizona State University, Tempe, AZ, USA, all in electrical engineering, in 2009, 2011, and 2015, respectively. Currently, he works with Maxlinear, Carlsbad, CA, USA. His research interests include various topics in information theory and wireless communications with a particular focus on coding techniques.

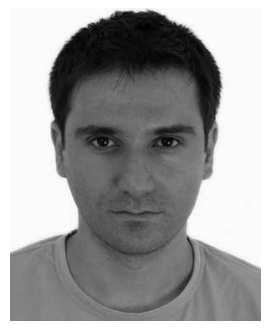
and multichannel processing.

A. Korhan Tanc (S'10-M'12) received the Ph.D. degree in electronics and communications engineering from Istanbul Technical University, Istanbul, Turkey, in 2011. From September 2012 to June 2013, he was a Postdoctoral Researcher with the School of Electrical, Computer, and Energy Engineering, Arizona State University, Tempe, AZ, USA. He is currently an Assistant Professor of Electrical and Electronics Engineering with Kirklareli University, Kirklareli, Turkey. His research interests include adaptive signal processing, communication theory,

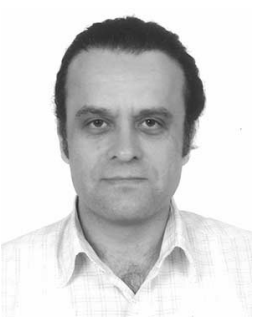

Tolga M. Duman (S'95-M'98-SM'03-F'11) received the B.S. degree from Bilkent University, Ankara, Turkey, the M.S. and Ph.D. degrees from Northeastern University, Boston, MA, USA, all in electrical engineering, in 1993, 1995, and 1998, respectively. Prior to joining Bilkent University in September 2012, he was with the Department of Electrical Engineering, Arizona State University, Tempe, AZ, USA, as an Assistant Professor (1998-2004), an Associate Professor (2004-2008), and a Professor (2008-2015). He is a Professor of Electrical and Electronics Engineering, Bilkent University, and an Adjunct Professor with the School of Electrical, Computer, and Energy Engineering, Arizona State University. His research interests include systems, with particular focus on communication and signal processing, including wireless and mobile communications, coding/modulation, coding for wireless communications, data storage systems, and underwater acoustic communications. He served as an Editor for the IEEE TRANSACTIONS ON WIRELESS COMMUNICATIONS (2003-2008), the IEEE TRANSACTIONS ON COMMUNICATIONS (20072012), and the IEEE ONLINE Journal OF SuRveys AND Tutorials (2002-2007). He is currently an Area Editor for the IEEE TRANSACTIONS ON COMMUNICATIONS (coding and communication theory) (2011-present) and an Editor for Physical Communications journal (Elsevier) (2010-present). He was a recipient of the National Science Foundation CAREER Award and the IEEE Third Millennium Medal. 